\title{
DEVELOPMENT OF WIRELESS MODULE FOR TUNNEL VIBRATING WIRE TYPE SENSOR
}

\author{
Jung Yeol, Kim \\ Hyun Suk, Yoo \\ Researcher \\ KICT, 2311, Daehwa-dong, \\ Ilsanseo-gu, Goyang-si, \\ Gyeonggi-do, 411-712, \\ South Korea \\ \{jrkim, hsyoo\}@kict.re.kr
}

\author{
Soon Wook, Kwon \\ Professor \\ Sungkyunkwan Univ., \\ 300 Cheoncheon-dong, \\ Jangan-gu, Suwon, Gyeonggi-do, \\ 440-746, South Korea \\ swkwon@skku.edu
}

\author{
Moon Young, Cho \\ Research Fellow \\ KICT, 2311, Daehwa-dong, \\ Ilsanseo-gu, Goyang-si, \\ Gyeonggi-do, 411-712, \\ South Korea \\ mycho@kict.re.kr
}

\begin{abstract}
Tunnel construction is being increased rapidly in Korea. About 60 percent of total tunnels have been constructed for the last 10 years. So systematic tunnel measurement and maintenance system have been needed. Vibrating wire type sensor is widely used in tunnel measurement system because of their stability and durability. Vibrating wire element is applied strain gauge, earth pressure cell, temperature gauge, piezometer and so forth. But conventional vibrating wire sensor is using wire-based communication and many problems have been occurred like high cost and disconnection. So we developed low-cost and effective wireless communication module for vibrating wire type sensor using pulse counting and Zigbee technologies. This module can change conventional wire-based data log and communication into wireless one instantly. This paper describes prototype of wireless communication based vibrating sensor system and results of field test.
\end{abstract}

\section{KEYWORDS}

Tunnel, Measurement, Zigbee, Wireless

\section{INTRODUCTION}

It has been steadily increasing tunnel construction since more than $70 \%$ of the nation consists of mountain region in Korea. The number of tunnels in national road has been growing for ten years[1]. And it was significantly increasing in highway. Therefore it is very important for monitoring to better accomplish tunnel construction and main- tenance, and a systematic monitoring plan should be carried out for acquisitioning useful and reliable field measurement information. However, the tunnel monitoring has been conducting in limited area and has not organized in acquisition data from sensors. So the measurement data from sensors has not used in construction and maintenance in real situation of tunnels. The reasons are that existing wired sensors are complicated for 
control and maintenance. Moreover expensive data logger has less economical feasibility. As a result it is import to development of sensors having economical feasibility, to effective sensing and control of sensors in tunnel maintenance. In this paper, Zigbee based wireless communication prototype module which could change current wired vibration wire sensor into wireless sensing device was introduced. Field tests and the results using developed wireless sensor module are also included.

\section{TUNNEL MEASUREMENT AND VIBRATING WIRE SENSOR}

Tunnel measurement for construction can be divided into 'daily management measurement(A type) and 'representative point measurement(B type)'. The daily management measurement is subject to all the construction site, which is to confirm the safety of the tunnel construction and include face mapping, lining displacement measurement, tunnel arch subsidence measurement, soil subsidence measurement. The representative point measurement is subject to a inspection of design, planning of construction and changing design and include underground displacement measurement, rock-bolt axial force measurement, shotcrete stress measurement, underground subsidence measurement [2][3].

Vibrating wire sensor is widely used in these measurements mentioned above. But they have problems of cost, usability and durability because they are wire based. Therefore, most engineers in tunnel construction site perform minimum level of measurement at necessary point.

\section{WIRELESS PROTOTYPE OF THE VIBRATING WIRE SESOR}

\subsection{Concept}

The wireless module for a vibrating wire sensor made in this study is connected to various types of vibrating wire sensor installed in the tunnel as shown in Fig. 1 in order to relay or transmit sensing data in a wireless method.

All the data in sensors to which the module is connected are ultimately transmitted to the notebook computer on the spot. Here, the notebook computer using Zigbee gateway and USB is used as the operating server for manufacturing the prototype in this study. CDMA communication module and LAN can be applied to the notebook computer in the event that measurement in a long distance is needed.

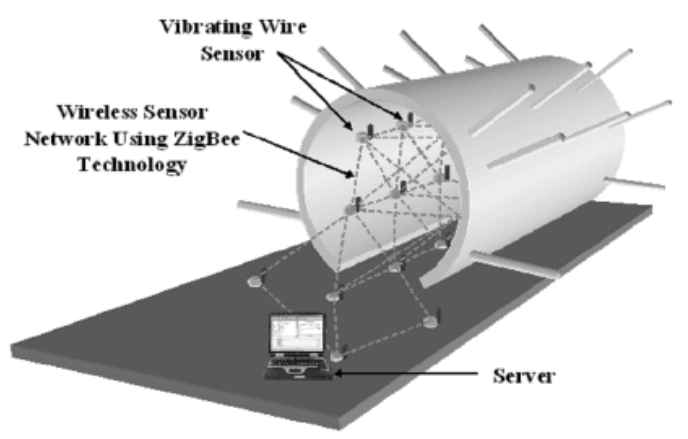

Figure 1 Concept of Wireless Tunnel Measurement

\subsection{Wireless Module}

The wireless sensor module consists of a vibrating wire $\mathrm{A} / \mathrm{D}$ circuit, a Zigbee communication circuit, a battery, packaging, operation software, and a notebook computer as shown in Figure 2.

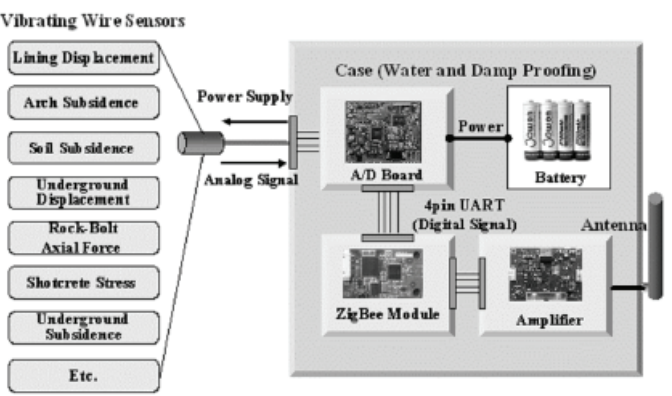

Figure 2 Composition of Wireless Module

Of the components, the vibrating wire $\mathrm{A} / \mathrm{D}$ circuit is a circuit to convert analog signals into digital pulse. The vibrating wire A/D circuit, which has an interface (4 pin) to be connected to sensors, provides sensors with electric power, changes the cycle and frequency of measurement, and conditions (filters or amplifies) analog signals as well as plays the basic function, $\mathrm{A} / \mathrm{D}$ converting. The vibrating wire $\mathrm{A} / \mathrm{D}$ circuit was manufactured by miniaturizing a conventional vibrating wire 
multi-channel data logger, so that it plays the same functions as a conventional logger.

Table 1 Specification of A/D Circuit

\begin{tabular}{|c|c|}
\hline Item & Specification \\
\hline Sensor & vibrating wire sensor \\
\hline Sensor Interface & 4 pin \\
\hline Zigbee Interface & 4 pin (UART) \\
\hline Frequency & $450 \sim 6,000 \mathrm{~Hz}$ \\
\hline Resolution & $0.1 \mathrm{~Hz}$ \\
\hline Accuracy & $0.01 \% \mathrm{FSR}$ \\
\hline Temperature & $-40 \sim 60^{\circ} \mathrm{C}$ \\
\hline Memory & $6,000 \mathrm{Read} /$ Record \\
\hline Power & $6 \mathrm{~V}(4 \mathrm{CR} 123 \mathrm{~A}$ Battery $)$ \\
\hline
\end{tabular}

Sensing data converted into digital pulse are transmitted to the Zigbee gateway through the Zigbee communication circuit using a RF (radio frequency) wireless method. The data received are saved in the notebook computer that works as a server on the spot, and displayed as graphs by the operation software. Detailed specification and prototype are as follows.

Figure 3 shows the prototype of wireless module for vibrating wire sensor. The module has two channels to connect sensors.

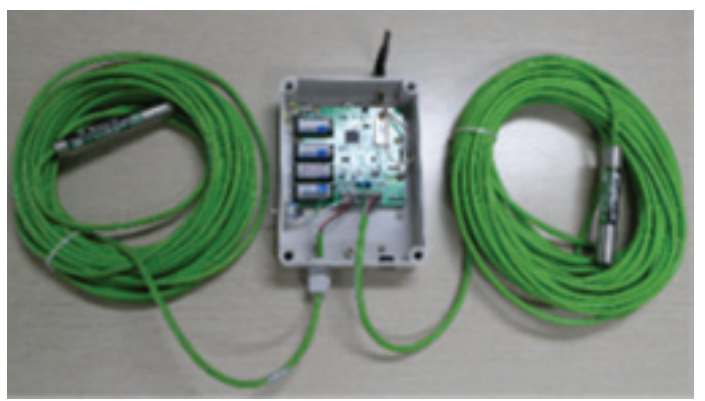

Figure 3 Wireless Module \& Vibrating

Wire Sensor

\subsection{Operation Software}

A notebook PC is used as a data server and connected to the Zigbee gateway using a USB port. The operation software is composed of application programs based on Microsoft Windows Operation System, and has the functions to automatically save and sort the data received from the Zigbee gateway in database(Figure 4). In addition, the data can be exported as a file format like a spreadsheet using MS Excel. Moreover, the operation software can display sensing data in graph format in a real time and adjust the sensing cycle between 1 and 86,400 seconds. The operation software has the function to check overall network status and uses the Mesh Networking, so that the data transmitting path can be automatically changed in the event of disconnection of a sensor (ad-hoc).

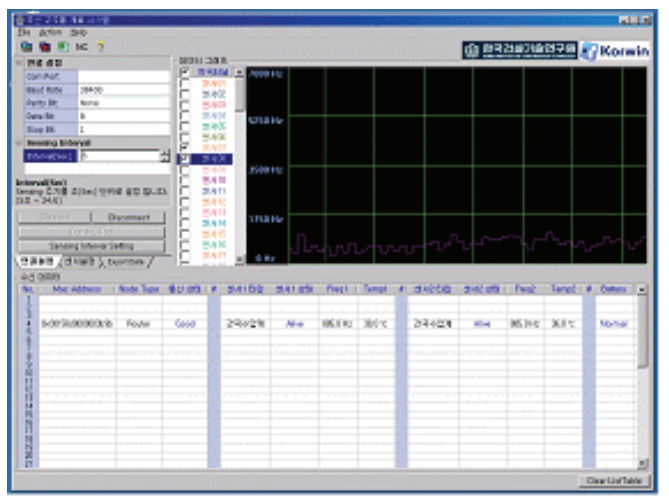

Figure 4 Operation Software

\section{FIELD TEST}

The filed test for the wireless module for vibrating wire sensor and operation software developed was performed in the Bukbyeon tunnel located in Gimpo-si(South Korea). This tunnel is being currently used, so that noise and dust are generated due to continuous vehicle access. The experiment was conducted in order to measure the maximum communication distance and verify smooth operation of the Mesh Network. At the outset, communication disorders were expected due to diffused reflection caused by vehicles, generation of interfering waves and wave absorption by the tunnel walls and the road surface. Unlike the expectation, the maximum communication distance 
is more than $200 \mathrm{~m}$ and the Mash Network works without difficulty. It is believed that the inside of the tunnel is made of nonconductor that plays a type of waveguide.

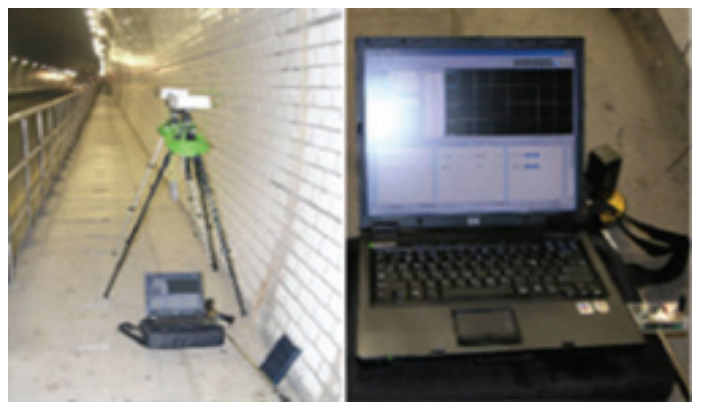

Figure 5 Field Test in Tunnel

\section{MEANINGS AND FUTURE IM- PROVEMENT}

\subsection{Distributed Data Logging in Tunnel}

A conventional tunnel measurement system has the central data logger and adopts the wired transmission method. This method is impossible to receive data from dozens of sensors connected to the logger in the event of disorders of the logger or the communication line. However, when the data logging is distributedly performed in each node and the communication is conducted using a Zigbee Mesh Network, there is almost no influence on the overall system in the event that several nodes do not work. Durability of communication will be possessed like the Internet. In addition, the conventional logger needs to be composed of a high-performance CPU, a memory, and a circuit to perform logging from dozens of sensors, and its cost is, therefore, expensive. However, when the distributed data logging is performed, the module can be manufactured at a low price.

\subsection{Future Improvement of Communica- tion Algorithm}

The wireless networking for the system currently developed is done for 24 hours to maintain the Mesh Network. It consumes a lot of electricity, and it can actually perform measurement only for at least 2 days up to maximum 5 days. This method can be used to perform a provisional measurement with a view to assessing safety of a tunnel currently used. Therefore, the algorithm should be revised for a maintenance measurement in a long term perspective.

The maintenance measurement in a tunnel is classified as the static measurement, and it is enough to carry out the measurement once a day. Therefore, each module waits in a sleep mode almost 24 hours and wakes up at a set time everyday to conduct measurement and communication for a certain period of time (5 minutes). For this method, time synchronization is important and the standard time is transmitted from the gateway connected to the server when all the modules wake up from the sleep mode, so that the time can be identically kept. If the method is used, the hardware with the battery currently used can arithmetically perform the measurement for at least 288 days up to maximum 1,440 days.

\subsection{Future Improvement of Data Man- agement System}

When the system is expanded and used in many tunnels (or nationwide), it is expected that a large volume of data should be verified, integrated, and managed in a real time in the future. To manage such a large volume of sensing data, a lot of middleware has been recently developed all over the world. ETRI (Electronics and Telecommunications Research Institute) in Korea developed middleware called Ubicore[4]. The middleware was developed to create or delete space for data to be saved, and perform sampling of sensing data, data analysis and inquiry by using XMLbased user designating context..

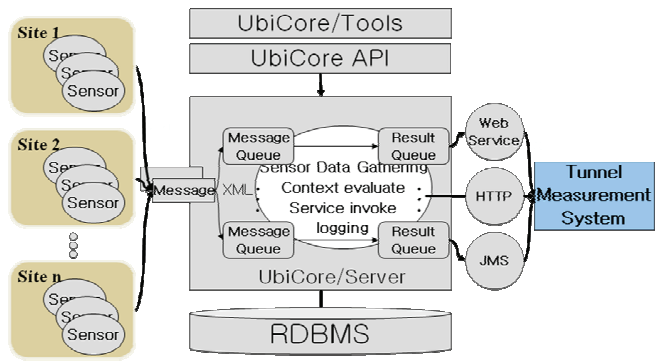

Figure 6 Concept of Data Management System 
The Ubicore will be used in relation with 'systematic management of the measured data and database system development' for this study, and Fig. 6 shows a system designed to process data from a lot of tunnels. Using the designated XML context, a lot of tunnels shown on the left of the Figure transmit sensing data, and Ubicore analyzes, arranges and saves the context in database. When a user requests data via query, Ubicore interprets query and fetch the data from the database using the web-service, HTTP, or JMS (Java Message Service).

\section{CONCLUSION}

There are many barriers for using sensors in tunnel such as noise, vibration, dust, humidity. The developed wireless communication module has significant meaning in using current vibration wire sensor by integrating with wireless communication function. Therefore, using this module, existing vibration wire type sensor can be easily embeded wireless communication function. Moreover, this module can be applied widely and improve applicability of sensors in tunnels and other social infrastructure system. In addition, field tests conducted by using this prototype module to verify its performance. The limitations found in field tests in current module including battery usage and sensing zone generation will be complimented.

\section{ACKNOWLEDGMENT}

This study is about the results of a base research of Korea Institute of Construction Technology, 'Development of Automatic Tunnel Monitoring System to Prevent Structural Failures \& Collapses

\section{REFERENCES}

[1] Ministry of Construction \& Transportation (2006) 2005 Bridge-Tunnel Records, Ministry of Construction \& Transportation, South Korea

[2] Kyu Jin Bae, Sang Joon Ma et al. (1998) Development of Rapid Electronic Railway Tunnel Technology, Ministry of Construction \& Transportation, South Korea

[3] Ministry of Construction \& Transportation (1999), Standard Specification of Tunnel, Ministry of Construction \& Transportation, South Korea

[4] M. J. Kim (2006), Mass Sensor Data Stream Processing Framework API Function Manual, Electronics and Telecommunication Research Institute. 